\title{
Alteration of Virulence and Aggressiveness during Serial Transfer and Simplified Storage of Rice Blast Fungus Pyricularia oryzae Cav.
}

\author{
Nobuaki Matsuyama* and Tomio Yamaguchi** \\ 松山宣明*・山富夫** : 継代保存中におけるイネいるち病菌 Pyricularia \\ oryzae Cav. の病原性，病原力の変化と簡易保存法の検討
}

\begin{abstract}
Serial transfer of Pyricularia oryzae on PSA slant during 4 years caused decrease of aggressiveness and loss or acquirement of virulence. Monoconidialy re-isolated lines from isolate which appeared as not to be varied were, in some cases, heterogeneous in virulence and/or aggressiveness. Alteration was affected by frequency of transferring, volume of culture medium and constituents of medium. Frequent renewal of medium was commoniy unfavorable. Although synthetic medium was better than PSA, the variation also occurred at 3 rd year of storage. Rice node method is better than serial transfer on PSA slant. Around $60-70 \%$ stocks, however, died or contaminated during storage in $-10 \mathrm{C}$ for 4-10 years. A simple and reliable method for preservation of this fungus was investigated. Dried mycelial discs were viable, at least, for 5 years of preservation in small vials desiccated with silica gel under 5 to $-10 \mathrm{C}$.
\end{abstract}

(Received February 2, 1981)

\section{Introduction}

The storage of microorganism without any change of their characteristics is most important subject and has long been studied. In phytopathological field, the maintenance of virulence and aggressiveness of isolates is most essential. Some experiments indicated that how virulence and aggressiveness of Pyricularia oryzae is unstable like other microorganisms during serial transferring ${ }^{3,4,9,10,12)}$ and how preservation on PSA medium is unreliable ${ }^{9,10)}$. There are several reports on preservation of properties of this fungus $1,7,15)$. Some methods, however, need special facilities and the other have various defects. In this report, a simple and reliable method developed by the authors will be presented. The terminology of virulence and aggressiveness was agreed with van der Plank ${ }^{16}$ ) and Watson ${ }^{17}$.

Most experiments were carried out at National Institute of Agricultural Sciences, Nishigahara (NIAS), Tokyo.

\section{Materials and Methods}

Every isolate of Pyricularia oryzae Cav. used in this experiment was monoconidialy

* Faculty of Agriculture, Kyushu University, Fukuoka 812, Japan 九州大学農学部

** National Institute of Agricultural Sciences, Tsukuba, Ibaraki 305, Japan 農業技術研究所 
isolated from diseased rice plant and had been maintained at NIAS. The change of virulence and/or aggressiveness of each isolate during storage was tested by inoculation to 12 differential varieties set ${ }^{5,8,8}$. Each isolate used for inoculation was cultured on oat meal medium ${ }^{11)}$ at $25 \mathrm{C}$ for 12 days. Aerial mycelia of colony were taken away with spatula at 10 th day and colony were kept for 2 days at $25 \mathrm{C}$ under fluorescent light or BLB lump for sporulation. Spores formed on a Petri dish were brushed off with $50 \mathrm{ml}$ of dist. water and its concentration was adjusted so as to contain 5-20 spores in $\times 150$ microscopic field. The suspension was sprayed with wetting agent $\left(T_{\text {ween }} 20\right)$ to the differential varieties. The inoculated plants were kept in moist chamber for about $12 \mathrm{hr}$ at $23-25 \mathrm{C}$. The symptoms were observed at 7-9 th day after inoculation.

Exp. 1. Forty-three isolates (Table 1) were successively cultured on PSA slant for 4 years. After routine inoculation, virulence and aggressiveness to differential varieties were compared with the original virulence and aggressiveness documented.

Exp. 2. Successive culturing on PSA slant is easiest way and often been used, but it caused frequently the decrease of aggressiveness and loss or acquirement of virulence. To prevent such variation of properties, favorable volume of culture medium, frequency of transferring and more suitable medium were tested as follows.

Ten isolates which were newly isolated from rice blast lesion collected from various places in Japan were cultured on $1.5 \mathrm{ml}$ of PSA medium in $6 \mathrm{ml}$ test tube(S) and on 15 $\mathrm{ml}$ of medium in $35 \mathrm{ml}$ test tube(L). Each isolate was successively transferred every 1 and 3 months for renewal of medium. Each of above cultures was designated and abbreviated as $1 \mathrm{~S}, 1 \mathrm{~L}, 3 \mathrm{~S}$ and $3 \mathrm{~L}$ respectively. Virulence and aggressiveness of isolates were tested after 2 years.

Seventeen isolates which were newly isolated from rice blast lesions collected from various places were respectively cultured on slants of three kinds of media in $6 \mathrm{ml}$ test tubes at $20 \mathrm{C}$ and serially transferred every 2 months. After 1 and 3 yesrs virulence and aggressiveness to 12 differential varieties were tested. The constituents of media were as follows. Synthetic medium: $3 \mathrm{~g} \mathrm{KNO}_{3}, 0.5 \mathrm{~g} \mathrm{KH}_{2} \mathrm{PO}_{4}, 0.5 \mathrm{~g} \mathrm{~K} \mathrm{HPO}_{4}, 0.5 \mathrm{~g} \mathrm{MgSO}$, $1 \mathrm{mg} \mathrm{CaCl}$, $1 \mathrm{mg} \mathrm{FeCl} 3,1 \mathrm{mg}$ thiamine, $5 \mu \mathrm{g}$ biotin, $20 \mathrm{~g}$ glucose, $18 \mathrm{~g}$ agar, $1,000 \mathrm{ml}$ dist. water, $\mathrm{pH}$ 5.7. Yeast extract added medium : $5 \mathrm{~g}$ yeast extract was added for $\mathrm{KNO}_{3}$ to synthetic medium stated above. Potato sucrose agar (PSA): $1,000 \mathrm{ml}$ of potato decoction, $20 \mathrm{~g}$ sucrose, $18 \mathrm{~g}$ agar.

Exp. 3. Hoku 373 and newly isolated Ken 68-01 were monoconidialy re-isolated at the start of this experiment and were successively cultured on PSA slant. After 18 months each isolate was cultured on oat meal medium ${ }^{11}$ in Petri dish for sporulation. Each colony was divided into four sections and five spores were monoconidialy isolated from each section. Monoconidial lines were cultured and sporulated respectively. Then their virulence and aggressiveness to differential varieties were tested as stated previously. The ability of sporulation of each line was tested before inoculation.

Exp. 4. Since virulence and aggressiveness of isolate varied even on synthetic medium, more reliable storing methods were needed.

Nineteen isolates were cultured on oat meal agar ${ }^{11}$, synthetic medium stated above and PSA medium. Plates of oat meal agar were developed under BLB lump for sporulation at final 2 days of culturing. For this purpose plastic Petri dish was suitable because of its perviousness to near ultra-violet light which is stimulative for sporulation of this 
fungus. After 10-12 days, margin of mycelial mat was cut with cork borer. Mycelial discs were transferred on sterilized paper disc $(1 \mathrm{~mm}$ in thickness, $8 \mathrm{~mm}$ in diameter, Toyo Seisakusho Co. Ltd.) and kept them in $25 \mathrm{C}$ until dryness. Mycelial discs were stuck on surface of paper disc. Then the discs were put in sterilized screw vials (73 mm in length, $18 \mathrm{~mm}$ in diameter) with silica gel. The amount of silica gel was one-third volume of the vial. Sterilization of vials and silica gel was conducted as follows. The cap of screw vial with gum packing was slightly loosed and then vial was autoclaved at $120 \mathrm{C}$ for $15 \mathrm{~min}$ and followed by drying at $100 \mathrm{C}$. Silica gel was dried and sterilized at $160 \mathrm{C}$.

Vials which involve samples were covered with vinyl bag and desiccated further by desiccant. These bags were kept in freezer $(-10 \mathrm{C})$ and refrigerator $(5 \mathrm{C})$. After 5 years, survival of each isolate was tested.

Since most experiments were conducted before the proposal of new differential varieties set ${ }^{18}$, former designation of races $^{5,6)}$ was used in this study.

\section{Results and Discussion}

Serial transfer on PSA medium is a easy way and has been commonly used for storage of fungi. However, maintenance of virulence and aggressiveness is not easy and sometimes it is difficult even for one month after isolation of pathogen from diseased plant. As shown in Table 1 , more than $79 \%$ isolates of Pyricularia oryzae lost or acquired virulence and decreased aggressiveness during storage for 4 years.

Table 1. Alteration of virulence and aggressiveness to 12 differential varieties during serial transfer on PSA slant for 4 years

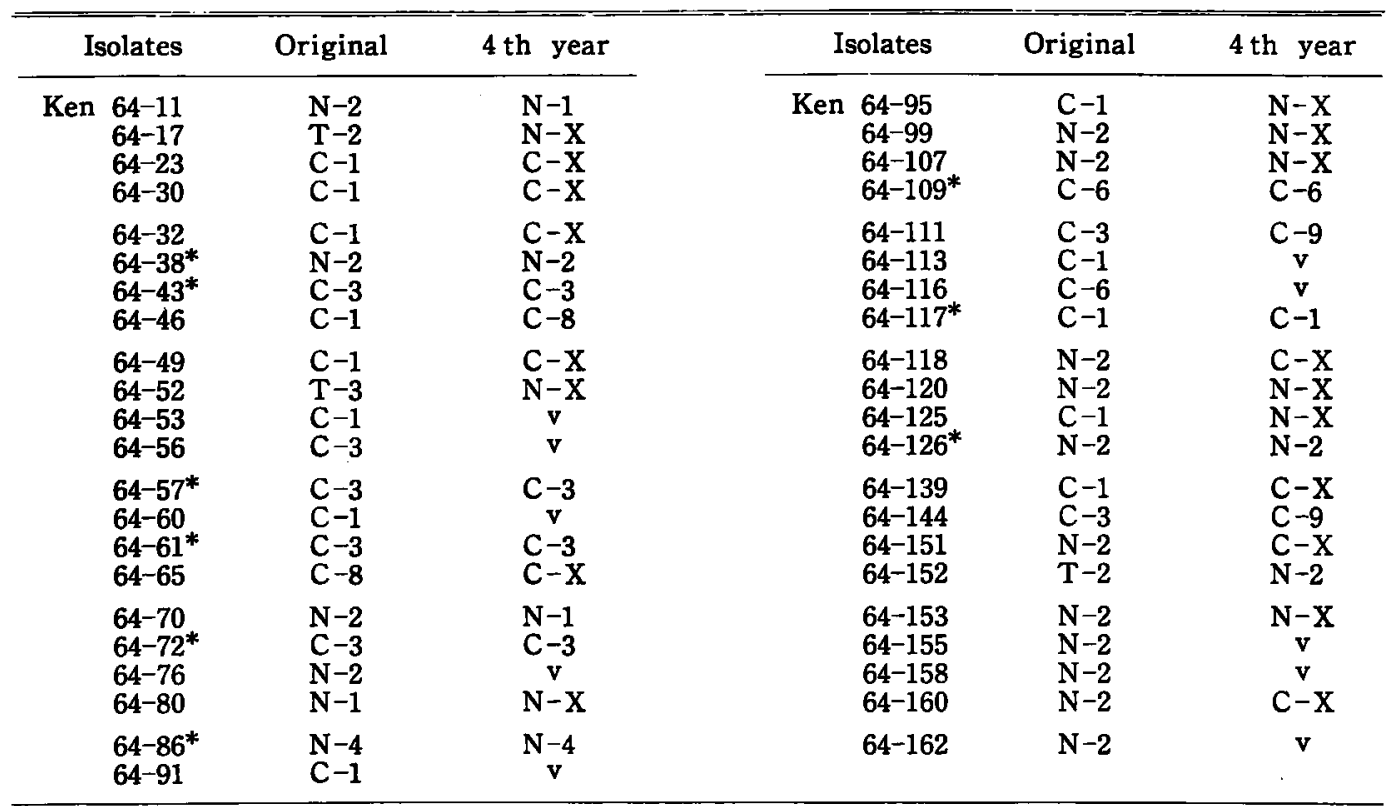

$\mathrm{C}-\mathrm{X}$ and $\mathrm{N}-\mathrm{X}$ mean new $\mathrm{C}$ and $\mathrm{N}$ race.

$v$ : Lost its virulence and/or aggressiveness.

*: Not varied.

Designation and abbreviation of races were agreed with the former style ${ }^{5,0)}$. 
Whether pathogenic alteration observed in this experiment is due to decrease of aggressiveness or loss of virulence is indefinite. Many variants formed relatively few and small lesions on every varieties infected. This result would indicate that pathogenic alteration during serial transfer may be mainly due to decrease of aggressiveness.

Ken 68-01 showed original virulence to differential varieties after 18 months of

Table 2. Heterogeneity of virulence and/or aggressiveness of monoconidialy re-isolated lines from isolates which appeared as not to be varied after serial transfer on PSA slant for 18 months

\begin{tabular}{|c|c|c|c|c|c|c|c|c|c|c|c|c|c|c|c|c|c|c|c|c|}
\hline \multirow{2}{*}{$\begin{array}{l}\text { Differential } \\
\text { varieties }\end{array}$} & \multicolumn{20}{|c|}{ Hoku $373(\mathrm{~N}-1)$} \\
\hline & $a-1$ & 2 & 3 & 4 & 5 & $b-1$ & 2 & 3 & 4 & 5 & $c-1$ & 2 & 3 & 4 & 5 & $d-1$ & 2 & 3 & 4 & 5 \\
\hline Te-tep & $\mathbf{R}$ & $\mathbf{R}$ & $\mathbf{R}$ & $\mathbf{R}$ & $\mathbf{R}$ & $\mathbf{R}$ & $\mathbf{R}$ & $\mathbf{R}$ & $\mathbf{R}$ & $\mathbf{R}$ & $\mathbf{R}$ & $\mathbf{R}$ & $\mathbf{R}$ & $\mathbf{R}$ & $\mathbf{R}$ & $\mathbf{R}$ & $\mathbf{R}$ & $\mathbf{R}$ & $\mathbf{R}$ & $\mathbf{R}$ \\
\hline Tadukan & $\mathbf{R}$ & $\mathbf{R}$ & $\mathbf{R}$ & $\mathbf{R}$ & $\mathbf{R}$ & $\mathbf{R}$ & $\mathbf{R}$ & $\mathbf{R}$ & $\mathbf{R}$ & $\mathbf{R}$ & $\mathbf{R}$ & $\mathbf{R}$ & $\mathbf{R}$ & $\mathbf{R}$ & $\mathbf{R}$ & $\mathbf{R}$ & $\mathbf{R}$ & $\mathbf{R}$ & $\mathbf{R}$ & $\mathbf{R}$ \\
\hline Usen & $\mathbf{R}$ & $\mathbf{R}$ & $\mathrm{R}$ & $\mathbf{R}$ & $\mathbf{R}$ & $\mathbf{R}$ & $\mathbf{R}$ & $\mathbf{R}$ & $\mathbf{R}$ & $\mathbf{R}$ & $\mathbf{R}$ & $\mathbf{R}$ & $\mathbf{R}$ & $\mathbf{R}$ & $\mathbf{R}$ & $\mathbf{R}$ & $\mathbf{R}$ & $\mathbf{R}$ & $\mathbf{R}$ & $\mathbf{R}$ \\
\hline Chokoto & $\mathbf{R}$ & $\mathbf{R}$ & $\mathbf{R}$ & $\mathbf{R}$ & $\mathbf{R}$ & $\mathbf{R}$ & $\mathbf{R}$ & $\mathbf{R}$ & $\mathbf{R}$ & $\mathbf{R}$ & $\mathbf{R}$ & $\mathbf{R}$ & $\mathbf{R}$ & $\mathbf{R}$ & $\mathbf{R}$ & $\mathbf{R}$ & $\mathbf{R}$ & $\mathbf{R}$ & $\mathbf{R}$ & $\mathbf{R}$ \\
\hline Yakeiko & $\mathbf{R}$ & $\mathbf{R}$ & $\mathbf{R}$ & $\mathbf{R}$ & $\mathbf{R}$ & $\mathbf{R}$ & $\mathbf{R}$ & $\mathbf{R}$ & $\mathbf{R}$ & $\mathbf{R}$ & $\mathbf{R}$ & $\mathbf{R}$ & $\mathbf{R}$ & $\mathbf{R}$ & $\mathbf{R}$ & $\mathbf{R}$ & $\mathbf{R}$ & $\mathbf{R}$ & $\mathbf{R}$ & $\mathbf{R}$ \\
\hline Kanto 51 & $\mathbf{R}$ & $\mathbf{R}$ & $\mathbf{R}$ & $\mathbf{R}$ & $\mathbf{R}$ & $\mathbf{R}$ & $\mathbf{R}$ & $\mathbf{R}$ & $\mathbf{R}$ & $\mathbf{R}$ & $\mathbf{R}$ & $\mathbf{R}$ & $\mathbf{R}$ & $\mathbf{R}$ & $\mathbf{R}$ & $\mathbf{R}$ & $\mathbf{R}$ & R 1 & $\mathbf{R}$ & $\mathbf{R}$ \\
\hline Ishikari shiroke & $\mathbf{S}$ & $\mathbf{S}$ & $\mathbf{S}$ & $\mathbf{S}$ & $\mathbf{R}$ & $\mathbf{S}$ & $\mathbf{s}$ & $\mathbf{S}$ & $\mathbf{S}$ & $\mathbf{S}$ & $\mathbf{S}^{*}$ & $\mathbf{R}$ & $\mathbf{S}^{*}$ & $\mathbf{S}$ & $\mathbf{S}$ & $\mathbf{S}$ & $\mathbf{S}$ & $\mathbf{S}$ & $\mathbf{S}$ & $\mathbf{S}$ \\
\hline Homarenishiki & $\mathbf{S}$ & $\mathbf{S}$ & $\mathbf{S}$ & $\mathbf{M}$ & $\mathbf{s}$ & $\mathbf{S}$ & $\mathbf{s}$ & $\mathbf{R}$ & $\mathbf{S}$ & $\mathbf{s}$ & $\mathbf{S}$ & $\mathbf{R}$ & $\mathbf{S}^{*}$ & $\mathbf{s}$ & $\mathbf{S}^{*}$ & $\mathbf{s}$ & $\mathbf{S}$ & $\mathbf{M}$ & $\mathbf{s}$ & $\mathbf{S}$ \\
\hline Ginga & $\mathbf{S}$ & $\mathbf{S}$ & $\mathbf{S}$ & $\mathbf{S}$ & $\mathbf{S}$ & $\mathbf{S}$ & $\mathbf{S}$ & $\mathbf{S}$ & $\mathbf{S}$ & $\mathbf{S}$ & $\mathbf{S}$ & $\mathbf{R}$ & $\mathbf{S}$ & $\mathbf{S}$ & $\mathbf{S}$ & $\mathbf{S}$ & $\mathbf{S}$ & $\mathbf{S}$ & $\mathbf{R}$ & $\mathbf{S}$ \\
\hline Norin No. 22 & $\mathbf{S}$ & $\mathbf{S}$ & $\mathbf{S}$ & $\mathbf{S}$ & $\mathbf{S}$ & $\mathbf{S}$ & $\mathbf{S}$ & $\mathbf{S}$ & $\mathbf{S}$ & $\mathbf{S}$ & $\mathbf{S}$ & $\mathbf{S}$ & $\mathbf{S}$ & $\mathbf{S}$ & $\mathbf{S}$ & $\mathbf{S}$ & $\mathbf{S}$ & $\mathbf{S}$ & $\mathbf{S}$ & $\mathbf{S}$ \\
\hline Aichi asahi & $\mathbf{S}$ & $\mathbf{S}$ & $\mathbf{S}$ & $\mathbf{S}$ & $\mathbf{S}$ & $\mathbf{S}$ & $\mathbf{S}$ & $\mathbf{S}$ & $\mathbf{S}$ & $\mathbf{S}$ & $\mathbf{S}$ & $\mathbf{S}$ & $\mathbf{S}$ & $\mathbf{S}$ & $\mathbf{s}$ & $\mathbf{S}$ & $\mathbf{S}$ & $\mathbf{S}$ & $\mathbf{S}$ & $\mathbf{S}$ \\
\hline Norin No. 20 & $\mathbf{S}$ & $\mathbf{S}$ & $\mathbf{S}$ & $\mathbf{S}$ & $\mathbf{S}$ & $\mathbf{S}$ & $\mathbf{S}$ & $\mathbf{S}$ & $\mathbf{S}$ & $\mathbf{S}$ & $\mathbf{S}$ & $\mathbf{S}$ & $\mathbf{S}$ & $\mathbf{S}$ & $\mathbf{s}$ & $\mathbf{S}$ & $\mathbf{S}^{*}$ & $\mathbf{S}$ & $\mathbf{S}$ & $\mathbf{S}$ \\
\hline Number of spores ${ }^{a)}$ & 12 & 13 & 8 & 8 & 9 & 14 & 8 & 11 & 9 & 13 & 20 & 11 & 23 & 121 & 11 & 13 & 9 & 111 & 12 & 5 \\
\hline \multirow{2}{*}{$\begin{array}{l}\text { Differential } \\
\text { varieties }\end{array}$} & \multicolumn{20}{|c|}{ Ken 68-01 (C-1) } \\
\hline & $a-1$ & 2 & 3 & 4 & 5 & $b-1$ & 2 & 3 & 4 & 5 & $c-1$ & 2 & 3 & 4 & 5 & $d-1$ & 2 & 3 & 4 & 5 \\
\hline Te-tep & $\mathbf{R}$ & $\mathbf{R}$ & $\mathbf{R}$ & $\mathbf{R}$ & $\mathbf{R}$ & $\mathbf{R}$ & $\mathbf{R}$ & $\mathbf{R}$ & $\mathbf{R}$ & $\mathbf{R}$ & $\mathbf{R}$ & $\mathbf{R}$ & $\mathbf{R}$ & $\mathbf{R}$ & $\mathbf{R}$ & $\mathbf{R}$ & $\mathbf{R}$ & $\mathbf{R}$ & $\mathbf{R}$ & $\mathbf{R}$ \\
\hline Tadukan & $\mathbf{R}$ & $\mathbf{R}$ & $\mathbf{R}$ & $\mathbf{R}$ & $\mathbf{R}$ & $\mathbf{R}$ & $\mathbf{R}$ & $\mathbf{R}$ & $\mathbf{R}$ & $\mathbf{R}$ & $\mathbf{R}$ & $\mathbf{R}$ & $\mathbf{R}$ & R & $\mathbf{R}$ & $\mathbf{R}$ & $\mathbf{R}$ & $\mathbf{R}$ & $\mathbf{R}$ & $\mathbf{R}$ \\
\hline Usen & $\mathbf{R}$ & $\mathbf{R}$ & $\mathbf{R}$ & $\mathbf{R}$ & $\mathbf{R}$ & $\mathbf{R}$ & $\mathbf{R}$ & $\mathbf{R}$ & $\mathbf{R}$ & $\mathbf{R}$ & $\mathbf{R}$ & $\mathbf{R}$ & $\mathbf{R}$ & R & $\mathbf{R}$ & $\mathbf{R}$ & $\mathbf{R}$ & R & $\mathbf{R}$ & $\mathbf{R}$ \\
\hline Chokoto & $\mathbf{S}$ & $\mathbf{R}$ & $\mathbf{R}$ & $\mathbf{S}$ & $\mathbf{s}$ & $\mathbf{R}$ & $\mathbf{S}$ & $\mathbf{s}$ & $\mathbf{S}$ & $\mathbf{S}$ & $\mathbf{R}$ & $\mathbf{R}$ & $\mathbf{R}$ & $\mathbf{R}$ & $\mathbf{R}$ & $\mathbf{R}$ & $\mathbf{R}$ & R & $\mathbf{R}$ & $\mathbf{R}$ \\
\hline Yakeiko & $\mathbf{S}^{*}$ & $\mathbf{R}$ & $\mathbf{R}$ & $\mathbf{S}$ & $\mathbf{S}$ & $\mathbf{R}$ & $\mathbf{S}$ & $\mathbf{S}$ & $\mathbf{S}$ & $\mathbf{S}$ & $\mathbf{R}$ & $\mathbf{R}$ & $\mathbf{R}$ & $\mathbf{R}$ & $\mathbf{R}$ & $\mathbf{R}$ & $\mathbf{R}$ & R $\mathbf{~}$ & $\mathbf{R}$ & $\mathbf{R}$ \\
\hline Kanto 51 & $\mathbf{S}$ & $\mathbf{R}$ & $\mathbf{R}$ & $\mathbf{S}$ & $\mathbf{S}$ & $\mathbf{R}$ & $\mathbf{S}$ & $\mathbf{S}$ & $\mathbf{S}$ & $\mathbf{S}$ & $\mathbf{R}$ & $\mathbf{R}$ & $\mathbf{R}$ & $\mathbf{R}$. & $\mathbf{R}$ & $\mathbf{R}$ & $\mathbf{R}$ & R & $\mathrm{R}$ & $\mathbf{R}$ \\
\hline Ishikari shiroke & $\mathbf{s}^{*}$ & $\mathbf{R}$ & $\mathbf{R}$ & $\mathbf{S}$ & $\mathbf{R}$ & $\mathbf{R}$ & $\mathbf{S}^{*}$ & $\mathbf{s}$ & $\mathbf{S}^{*}$ & $\mathbf{S}^{*}$ & $\mathbf{R}$ & $\mathbf{R}$ & $\mathbf{R}$ & R & $\mathbf{R}$ & $\mathbf{R}$ & $\mathbf{R}$ & $\mathbf{R}$ & $\mathbf{R}$ & $\mathbf{R}$ \\
\hline Homarenishiki & $\mathbf{R}$ & $\mathbf{R}$ & $\mathbf{R}$ & $\mathbf{S}$ & $\mathbf{R}$ & $\mathbf{R}$ & $\mathbf{s}^{*}$ & $\mathbf{S}$ & $\mathbf{R}$ & $\mathbf{R}$ & $\mathbf{R}$ & $\mathbf{R}$ & $\mathbf{M}$ & R & $\mathbf{R}$ & $\mathbf{R}$ & $\mathbf{R}$ & $\mathbf{R}$ & $\mathbf{R}$ & $\mathbf{R}$ \\
\hline Ginga & $\mathbf{S}$ & $\mathbf{R}$ & $\mathbf{R}$ & $\mathbf{S}$ & $\mathbf{S}$ & $\mathbf{R}$ & $\mathbf{S}$ & $\mathbf{s}$ & $\mathbf{S}$ & $\mathbf{S}$ & $\mathbf{R}$ & $\mathbf{R}$ & $\mathbf{R}$ & R & $\mathbf{R}$ & $\mathbf{R}$ & $\mathbf{R}$ & $\mathbf{R}$ & $\mathbf{R}$ & $\mathbf{R}$ \\
\hline Norin No. 22 & $\mathbf{S}$ & $\mathbf{R}$ & $\mathbf{R}$ & $\mathbf{S}$ & $\mathbf{R}$ & $\mathbf{R}$ & $\mathbf{S}$ & $\mathbf{s}$ & $\mathbf{S}$ & $\mathbf{s}$ & $\mathbf{R}$ & $\mathbf{R}$ & $\mathbf{R}$ & R & $\mathbf{R}$ & $\mathbf{R}$ & $\mathbf{R}$ & $\mathbf{R}$ & $\mathbf{R}$ & $\boldsymbol{R}$ \\
\hline Aichi asahi & $\mathbf{R}$ & $\mathbf{R}$ & $\mathbf{R}$ & $\mathbf{S}$ & $\mathbf{S}^{*}$ & $\mathbf{R}$ & $\mathbf{S}$ & $\mathbf{S}$ & $\mathbf{S}$ & $\mathbf{S}$ & $\mathbf{R}$ & $\mathbf{R}$ & $\mathbf{R}$ & R & $\mathbf{R}$ & $\mathbf{R}$ & $\mathrm{R}$ & $\mathbf{R}$ & & $\mathbf{R}$ \\
\hline Norin No. 20 & $\mathbf{R}$ & $\mathbf{R}$ & $\mathbf{R}$ & $\mathbf{S}$ & $\mathbf{S}^{*}$ & $\mathbf{R}$ & $\mathbf{S}$ & $\mathbf{S}$ & $\mathbf{S}$ & $\mathbf{S}$ & $\mathbf{R}$ & $\mathbf{R}$ & $\mathbf{R}$ & R & $\mathbf{R}$ & $\mathbf{R}$ & $\mathbf{R}$ & $\mathbf{R}$ & & $\mathbf{R}$ \\
\hline Number of spores ${ }^{a)}$ & 3 & 2 & 3 & 6 & 3 & 5 & 6 & 10 & 12 & 11 & 17 & 3 & 6 & 4 & 6 & 6 & 5 & 6 & 4 & 6 \\
\hline
\end{tabular}

$S^{*}$ : Quite few susceptible lesions were formed.

Colonies were divided to 4 sections and designated as a, b, c, d. Five spores were respectively isolated from each section.

a) Number of spores under $\times 150$ microscopic field. Spores formed on a plate were suspended to 50 $\mathrm{ml}$ of dist. water. 
successive culturing. However, monoconidialy re-isolated lines showed various virulence and/or aggressiveness (Table 2). While Hoku 373 which was isolated in 1959 and selected as a standard isolate was relatively homogeneous. These results indicate that frequency of alteration of virulence and aggressiveness is different according to isolate. The re-isolated lines from $b$ section of Ken 68-01 showed relatively stable virulence and aggressiveness. The correlation between these properties and sporulation ability was observed.

Iwata $^{8)}$ referred that frequent successiveculturing causes variation. In this experiment, long interval of serial transfer at every 3 months on $15 \mathrm{ml}$ culture-slant was better than others (Table 3 ). Recently a silicon plug for culure tube has been devised and this plug will be useful for long term preservation without frequent transferring. Since maintenance without any change of characteristics on PSA medium is difficult, more suitable media were examined. As shown in Table 4, 16 in 17 isolates tested were stable on synthetic medium at first year. However, variation also occurred even on synthetic medium at 3rd year though it was still better than other media. The authors reported that nucleic acid in culture medium could affect on variation ${ }^{2)}$ and this might be a reason why the synthetic medium was better.

It is well-documented that most fungi and bacteria survive under low temperature and/or desiccated condition. Anderson et al. ${ }^{2)}$ reported that $P$. oryzae survived under such adverse condition. This property has been utilized for storage of this fungus. The rice node method has been used by us for long-term preservation. However, this method has some defects. As shown in Table 5, around 60-70\% isolates died and contaminated during storage for $4-10$ years. Further, preparation of this medium is slightly troublesome. Therefore, more reliable
Table 3. Effect of frequency of serial transferring and volume of PSA slant on alteration of virulence and/or aggressiveness during 2 years culture

\begin{tabular}{rcccc}
\hline Isolates & $1 \mathrm{~S}$ & $1 \mathrm{~L}$ & $3 \mathrm{~S}$ & $3 \mathrm{~L}$ \\
\hline Ken $69-01$ & $\mathrm{v}$ & st & st & st \\
$69-02$ & v & v & v & st \\
$69-03$ & v & st & pv & pv \\
$69-04$ & st & v & st & st \\
$69-05$ & pv & st & v & st \\
$69-06$ & pv & pv & v & st \\
$69-07$ & v & v & v & pv \\
$69-08$ & v & v & pv & v \\
$69-09$ & pv & st & v & st \\
$69-10$ & v & pv & v & pv \\
\hline
\end{tabular}

The abbreviation $1 \mathrm{~S}, 1 \mathrm{~L}, 3 \mathrm{~S}$ and $3 \mathrm{~L}$ were explained in text.

$\mathrm{v}$ : Varied. Virulence and/or aggressiveness were completely lost.

pv : Partially varied. Virulence and/or aggressiveness to some differential varieties were varied.

st : Stable. No variation was observed.

Virulence and aggressiveness were tested on 12 differential varieties set.

Table 4. Alteration of virulence and aggressiveness to 12 differential varieties during successive culturing for 3 years on slants of various media

\begin{tabular}{|c|c|c|c|c|c|c|c|}
\hline \multirow{2}{*}{\multicolumn{2}{|c|}{ Isolates }} & \multicolumn{3}{|c|}{1 st year } & \multicolumn{3}{|c|}{3 rd year } \\
\hline & & Sy & Sy+YE & PSA & Sy & $\mathrm{Sy}+\mathrm{YE}$ & PSA \\
\hline \multirow[t]{17}{*}{ Ken } & $68-01$ & $\overline{\text { st }}$ & pv & st & st & $\mathbf{v}$ & $\mathbf{v}$ \\
\hline & $68-02$ & st & pv & st & $\mathbf{v}$ & $\mathbf{v}$ & st \\
\hline & $68-03$ & st & pv & st & st & $\mathbf{v}$ & st \\
\hline & $68-04$ & st & $\mathbf{v}$ & $\mathbf{v}$ & st & $\mathbf{v}$ & $\mathbf{v}$ \\
\hline & $68-05$ & st & $\mathbf{v}$ & $\mathbf{v}$ & $\mathbf{v}$ & $\mathbf{v}$ & $\mathbf{v}$ \\
\hline & $68-06$ & st & st & st & st & st & $\mathbf{v}$ \\
\hline & $68-07$ & st & st & st & $\mathbf{v}$ & $\mathrm{pv}$ & $\mathrm{pv}$ \\
\hline & $68-08$ & st & st & pv & $\mathbf{v}$ & st & $\mathbf{v}$ \\
\hline & $68-09$ & st & pv & $\mathbf{v}$ & st & $\mathbf{v}$ & $\mathbf{v}$ \\
\hline & $68-10$ & st & st & st & st & $\mathbf{v}$ & $\mathbf{v}$ \\
\hline & $68-11$ & st & st & st & st & st & st \\
\hline & $68-12$ & st & $\mathbf{v}$ & st & st & $\mathbf{v}$ & st \\
\hline & $68-13$ & st & st & st & st & $\mathbf{v}$ & st \\
\hline & $68-14$ & st & st & st & $\mathbf{v}$ & $\mathbf{v}$ & st \\
\hline & $68-15$ & $\mathbf{v}$ & $\mathbf{v}$ & st & $v$ & $\mathbf{v}$ & $\mathbf{v}$ \\
\hline & $68-16$ & st & st & $\mathbf{v}$ & st & st & $\mathbf{v}$ \\
\hline & $68-17$ & st & pv & pv & st & $\mathbf{v}$ & $\mathrm{v}$ \\
\hline \multicolumn{2}{|c|}{$\begin{array}{l}\text { Number of } \\
\text { stable isolate }\end{array}$} & 16 & 8 & 11 & 11 & 4 & 6 \\
\hline
\end{tabular}

Sy: Synthetic medium.

Sy+YE: Yeast extract was added to synthetic medium instead of $\mathrm{KNO}_{8}$.

PSA : Potato sucrose agar.

Abbreviation v, pv and st were explained in Table 3. 
Table 5. Survival of isolates which were stored by rice node method for $4-10$ years at $-10 \mathrm{C}$

\begin{tabular}{lccc}
\hline \multicolumn{1}{c}{ Viability } & 4 th year & \multicolumn{2}{c}{10 th year } \\
\hline Survived & 40 & 62 & 67 \\
Died (Contaminated) & $63(22)$ & $169(51)$ & $143(86)$ \\
\hline Total & 103 & 231 & $210^{2}$ \\
\hline
\end{tabular}

a) Foreign isolates from Philippines, Indonesia, Thailand, Vietnam, India, Taiwan, Hong Kong, Korea, Australia, Guinea and USA.

Table 6. Preservation of dried mycelial disc for 5 years under low temperature in vial desiccated with silica gel

\begin{tabular}{lccccccc}
\hline \hline & & \multicolumn{3}{c}{$5 \mathrm{C}$} & & \multicolumn{3}{c}{$-10 \mathrm{C}$} \\
\cline { 2 - 4 } \cline { 5 - 6 } Isolates & Oat & PSA & Sy & & Oat & PSA & Sy \\
\hline Hoku 373 & + & + & + & + & + & + \\
Ina 168 & + & + & + & + & + & + \\
Naga 87 & + & con. & + & + & + & + \\
Ken 53-11 & + & + & + & + & + & + \\
Ken 54-04 & + & + & + & + & + & + \\
Ken 54-20 & + & + & + & + & + & + \\
Ken 59-49 & con. & + & + & con. & + & + \\
Ken 60-19 & + & + & + & + & + & + \\
Ken 61-14 & + & + & + & + & + & + \\
Ken 62-03 & + & + & + & + & + & + \\
Ken 62-42 & + & + & + & + & + & + \\
Ken 62-76 & + & + & + & + & + & + \\
Ken 62-80 & + & + & + & + & + & + \\
Ken 66-20 & + & + & + & + & + & + \\
Ken 68-02 & + & + & + & + & + & + \\
TH 65-252 & + & + & + & + & + & + \\
TH 67-25 & + & + & + & + & + & + \\
Hiro 63-100 & + & + & + & + & + & + \\
Hiro 65-182 & + & + & + & + & + & + \\
\hline
\end{tabular}

Oat: Oat meal agar. ${ }^{11}$ Sporulation occured under BLB lump.

PSA : Potato sucrose agar. No sporulation occurred.

Sy: Synthetic medium. Sporulation occurred in darkness.

con. : Contaminated.

+ : Survived for 5 years.

Each isolate was cultured on various media respectively. Then, mycelial colony was cut with cork borer and dried.

and simple method was needed. Dried mycelial discs survived, at least, for 5 years in small vials desiccated with silica gel under low temperature (Table 6). It has been reported that the storage of spores is favorable for the preservation under low temperature. In this experiment, preservation with mycelial disc from PSA medium on which no sporulation occurred was also available. As shown previously, $70 \%$ stocks died in rice node method. All survived stocks, however, maintained perfectly their original virulence and aggressiveness under $-10 \mathrm{C}$. This result indicates that virulence and aggressiveness of 
all stocks preserved by this "Dried mycelial disc method" might also be well-maintained. Preliminarily sterilized sample-stock tube (commercially available) may be convenient for this method.

Authors are grateful to Prof. Dr. T. Kozaka, Tokyo University of Agriculture and Technology, for his many advices and Mrs. S. Ezaki for her assistance.

\section{Literature cited}

1. Akai, S. and Asada, T. (1961). Ann. Phytopath. Soc. Japan $26: 38-39$.

2. Anderson, A. L., Henry, B. W. and Morgan, T. L. (1948). Phytopathology 38 : 574.

3. Chien, C. C. (1968). J. Taiwan Agric. Res. 17: 22-29.

4. Giatogong, P. and Frederiksen, R. A. (1969). Phytopathology $59: 1152-1157$.

5. Goto, K. et al. (1967). Ann. Phytopath. Soc. Japan 33 (Extra issue) : 1-87.

6. Goto, K. and Yamanaka, S. (1968). Bull. Coll. Agric. Utsunomiya Univ. $7: 27-71$.

7. Hwang, S-W. (1966). Appl. Microbiol. 14: 784-788.

8. Iwata, K. (1977). Biseibutsu no Hozonho (Nei, T. ed.). Tokyo Univ. Press., Tokyo. p. 267.

9. Matsuyama, N. and Kozaka, T. (1970). Ann. Phytopath. Soc. Japan 36: 156.

10. Matsuyama, N. and Yamaguchi, T. (1980). Proc. Assoc. Pl. Prot. Kyushu $26: 177$.

11. Sekiguchi, Y. and Furuta, T. (1966). Ann. Phytopath Soc. Japan $32: 67$.

12. Shimoyama, M. (1960). Proc. Assoc. Pl. Prot. Hokuriku 8: 79-82.

13. Sleesman, J. P., Larsen, P. O. and Safford, J. (1974). Plant Dis. Reptr. 58 : 334-336.

14. Takahashi, Y. (1956). Ann. Phytopath. Soc. Japan $21: 136$.

15. Tominaga, T., Matsuyama, N. and Yamada, M. (1971). Ann. Phytopath. Soc. Japan $37: 368$.

16. Van der Plank, J. E. (1968). Disease Resistance in Plants. Academic Press, New York and London. p. 206.

17. Watson, I. A. (1970). Ann. Rev. Phytopathol. 8: 209-230.

18. Yamada, M., Kiyosawa, S., Yamaguchi, T., Hirano, T., Kobayashi, T., Kushibuchi, K. and Watanabe, S. (1976). Ann. Phytopath. Soc. Japan 42: 216-219.

19. Yamazaki, Y. and Kozaka, T. (ed.) (1980). Ineimochibyo to Teikoseiikushu. Hakuyu-sha, Tokyo. pp. 367-384.

和 文 摘 要

\section{継代保存中におけるイネいもち病菌 Pyricularia oryzae}

Cav.の病原性，病原力の変化と簡易保存法の検討

\section{松山宣明・山口富夫}

いもち病菌は PSA培地上で継代保存すると比較的短期間に病原力を低下させるか，病原性に変化を起こす (79\%)。保存中病原力に変化が無かったとみられる菌株でる，その単胞子再分離系統には可成りの変化がみ られる場合が多い。これらの性質の変化は培地，培地量，植継ぎ間隔などの培養条件の影䇺を受け易い。合 成培地は天然培地化比一病原力, 病原性の变化を起こしにくい。しかし, 保存後 3 年目には合成培地保存の すのにる変化がみられる。又培地量を多くし，植継ぎ間隔を出来るたけ長くすることも有効とみられる。一 方，節保存法は有用な一方法之考えられるが，低温保存中における雑菌の混入，死娍か多い。節保存 4 年目 に㧍ける死亡率は約60\%であり，10年目における死亡率は約 $70 \%$ であった。より簡易で確実な方法として乾

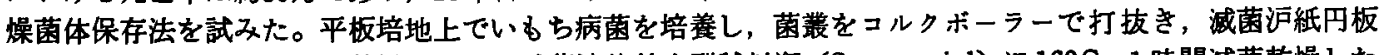
上に乗せシャーレ中で自然乾燥させる。減菌済蓋付小型試料瓶 (Screw vial) に160C，1時間隇菌乾燥した シリカゲルを入れ，その上に乾燥菌体の付着した汇紙円板数個を入れ密栓する。これらの操作は無菌的に行

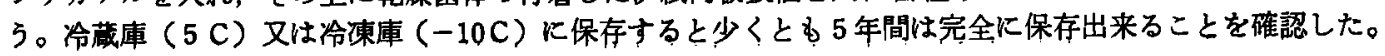

